\title{
On the Existence of Coincidence and Common Fixed Point of Two Rational Type Contractions and an Application in Dynamical Programming
}

\author{
Ahmed H. Soliman ${ }^{1}$ and Tamer Nabil ${ }^{2}$ \\ ${ }^{1}$ Department of Mathematics, Faculty of Science, Al-Azhar University, Assiut 71524, Egypt \\ ${ }^{2}$ Department of Basic Science, Faculty of Computers and Informatics, Suez Canal University, Ismailia, Egypt
}

Correspondence should be addressed to Ahmed H. Soliman; mahmod@kku.edu.sa

Received 29 March 2016; Revised 23 May 2016; Accepted 12 June 2016

Academic Editor: Pasquale Vetro

Copyright (C) 2016 A. H. Soliman and T. Nabil. This is an open access article distributed under the Creative Commons Attribution License, which permits unrestricted use, distribution, and reproduction in any medium, provided the original work is properly cited.

We establish some coincidence point results for self-mappings satisfying rational type contractions in a generalized metric space. Presented coincidence point theorems weaken and extend numerous existing theorems in the literature besides furnishing some illustrative examples for our results. Finally, our results apply, in particular, to the study of solvability of functional equations arising in dynamic programming.

\section{Introduction}

Banach contraction principle is one of the most important aspects of fixed point theory as a source of the existence and uniqueness of solutions of many problems in various branches inside and outside mathematics (see, [1-3]). Some generalizations of this theorem replace the contraction condition by a weaker. For instance, in 1975, Dass and Gupta [4] defined the following rational type contraction which is more general than the contraction condition:

$$
\begin{array}{r}
d(A x, A y) \leq a d(x, y)+\frac{b d(y, A y)(d(x, A x)+1)}{1+d(x, y)} \\
\forall x, y \in X, a, b \geq 0, a+b<1,
\end{array}
$$

where $A: X \rightarrow X$ is a mapping from a metric space $X$ into itself.

Recently, in 2015, Almeida et al. [5] introduced an extension of condition (1) of Dass and Gupta [4] as follows:

$$
\begin{array}{r}
d(A x, A y) \leq \phi(M(x, y))+C \\
\cdot \min \{d(x, A x), d(y, A y), d(x, A y), d(y, A x)\} \\
\forall x, y \in X, C \geq 0,
\end{array}
$$

where $M(x, y)$ is defined by

$$
\begin{aligned}
& M(x, y)=\max \left\{d(x, y), \frac{d(x, A x)(d(y, A y)+1)}{1+d(x, y)},\right. \\
& \left.\frac{d(y, A y)(d(x, A x)+1)}{1+d(x, y)}\right\}
\end{aligned}
$$

and $\phi:[0, \infty) \rightarrow[0, \infty)$ is a nondecreasing upper semicontinuous function with $\phi(t)<t$ for all $t>0$.

Another offshoot of generalizations of Banach's theorem is based on extending the axioms of metric spaces. It is worth mentioning that the use of triangle inequality in a metric space $(X, d)$ is of extreme importance, since it implies that $d$ is continuous; each open ball is an open set, a sequence may converge to unique point, and every convergent sequence is a Cauchy sequence and other things. In 2000, Branciari [6] introduced a new concept of generalized metric space by replacing the triangle inequality of a metric space by a socalled rectangular inequality. Since then, various works have dealt with fixed point results in such spaces (see, [7-16]). It was not directly noted that such generalized metric spaces (GMS, for short) may fail to satisfy the conditions which were mentioned above in metric spaces. 
In this paper, we introduce coincidence point theorems for two contraction self-mappings of rational type in generalized metric spaces. Our results improve the results of Almeida et al. [5]. These theoretical theorems are applied to the study of the existence solutions to a system of functional equations in dynamic programming.

\section{Preliminaries}

In this section, we present some preliminaries and notations related to rational type contraction and GMS.

Definition 1 (Branciari [6]). Suppose that $X$ be a nonempty set and $d: X \times X \rightarrow[0, \infty)$ be a distance function such that for all $w, x, y, z \in X$ and $w \neq x \neq y \neq z$,

(i) $d(w, x)=0 \Leftrightarrow w=x$,

(ii) $d(w, x)=d(x, w)$,

(iii) $d(w, x) \leq d(x, y)+d(y, z)+d(z, w)$ (quadrilateral inequality).

Then we called $(X, d)$ GMS.

The following example shows that GMS are more general than metric spaces.

Example 2. Suppose that $X=\{5 / 6,2 / 3,7 / 12,8 / 15\}$. Define $d$ on $X \times X$ as follows:

$$
\begin{aligned}
d\left(\frac{5}{6}, \frac{2}{3}\right) & =d\left(\frac{7}{12}, \frac{8}{15}\right)=\frac{4}{9} \\
d\left(\frac{5}{6}, \frac{8}{12}\right) & =d\left(\frac{2}{3}, \frac{7}{12}\right)=\frac{1}{3} \\
d\left(\frac{5}{6}, \frac{7}{12}\right) & =d\left(\frac{2}{3}, \frac{8}{12}\right)=\frac{8}{9} \\
d(x, x) & =0 \\
d(x, y) & =d(y, x) .
\end{aligned}
$$

Then $(X, d)$ is a GMS but not metric space.

Definition 3 (see $[6,17])$. Suppose that $(X, d)$ be a GMS and let $\left\{x_{n}\right\}$ be a sequence in $X$. Then

(i) $\left\{x_{n}\right\}$ converges to $x \in X$ in GMS if $\lim _{n \rightarrow \infty} d\left(x_{n}, x\right)=$ 0 ,

(ii) $\left\{x_{n}\right\}$ is a Cauchy in GMS if, $\forall \epsilon>0, \exists K(\epsilon)>0$ such that $d\left(x_{r}, x_{s}\right)<\epsilon, \forall r, s>K(\epsilon)$,

(iii) $(X, d)$ is called complete GMS if every Cauchy sequence in $X$ converges to a point in $X$.

Remark 4 (Sarma et al. [18]). Definition 1 of GMS does not ensure the following properties:

(a) $d$ is continuous on its domain.

(b) A GMS is Hausdorff. (c) There is a unique limit of a convergence sequence.

(d) Any convergent sequence is a Cauchy sequence.

In 2009, Samet [19] and Sarma et al. [18] introduced the following example which shows Remark 4.

Example 5 (see $[18,19])$. Suppose that $X=D \cup E$, where $D=$ $\{0,2\}$ and $E=\{1 / n: n=1,2,3, \ldots\}$. Define $d$ from $X \times X$ into $[0,+\infty)$ as follows:

$$
d(u, v)= \begin{cases}0, & u=v \\ 1, & u \neq v,\{u, v\} \subset D \text { or }\{u, v\} \subset E\end{cases}
$$

and $d(u, v)=d(v, u)=u$ if $u \in D$ and $v \in E$.

Then $(X, d)$ is a complete GMS. Moreover, one can see that

(1) $d(1 / n, 0)=0$ and $d(1 / n, 2)=2 \Rightarrow\{1 / n\}$ is not Cauchy sequence,

(2) there is no $r>0$ such that $B_{r}(0) \cap B_{r}(2)=\phi$; hence, GMS is not Hausdorff with the respective topology, where $B_{r}(a)=\{y \in X: d(a, y)<r, r>0\}$,

(3) $d(1 / n, 1 / 2) \neq d(1 / 2,0) \Rightarrow d$ is not continuous.

Lemma 6 (see [16]). Any Cauchy sequence in GMS converges to a unique point.

Definition 7 (see [17]). Let $A, B: X \rightarrow X$ and $\beta: X \times X \rightarrow$ $[0, \infty)$. The mapping $A$ is $B-\beta$-admissible if, for all $x, y \in X$ such that $\beta(B x, B y) \geq 1$, we have $\beta(A x, A y) \geq 1$. If $B$ is the identity mapping, then $A$ is called $\beta$-admissible.

Definition 8 (see [17]). Let $(X, D)$ be a GMS and $\beta: X \times X \rightarrow$ $[0, \infty) . X$ is $\beta$-regular if, for each sequence $\left\{x_{n}\right\}$ in $X$ such that $\beta\left(x_{n}, x_{n+1}\right)>1$ for all $n \in N$ and $x_{n} \rightarrow x$, there exists a subsequence $\left\{x_{n_{k}}\right\}$ of $\left\{x_{n}\right\}$ such that $\beta\left(x_{n_{k}}, x\right)>1 \forall k \in N$.

Definition 9 (see [17]). Suppose that $A, B: X \rightarrow X$ be two mappings from a nonempty set $X$ into itself. The mappings $A, B$ are said to be weakly compatible if $A t=B t, t \in X$ implies $A B t=B A t$. A point $z \in X$ is called point of coincidence of $A$ and $B$ if there exists a point $t \in X$ such that $z=A t=B t$.

\section{Main Results}

In this section we introduce some coincidence point results for two rational contraction self-mappings on GMS.

Theorem 10. Let $(X, d)$ be a GMS and let $A$ and $B$ be selfmappings on $X$ such that $A X \subset B X$. Suppose that $(B X, d)$ is a complete GMS and the following condition holds:

$$
\begin{aligned}
& d(A x, A y) \leq \phi(M(x, y))+C \min \{d(B x, A x), \\
&d(B y, A y), d(B x, A y), d(B y, A x)\} \\
& \forall x, y \in X, C \geq 0,
\end{aligned}
$$


where $M(x, y)$ is defined by

$$
\begin{gathered}
M(x, y)=\max \{d(B x, B y), \\
\frac{d(B x, A x)(d(B y, A y)+1)}{1+d(B x, B y)}, \\
\left.\frac{d(B y, A y)(d(B x, A x)+1)}{1+d(B x, B y)}\right\}
\end{gathered}
$$

and $\phi:[0,+\infty) \rightarrow[0,+\infty)$ is a continuous nondecreasing function and $\phi(t)=0 \Leftrightarrow t=0$.

Then $A$ and $B$ have a unique point of coincidence in $X$. Moreover, if $A$ and $B$ are weakly compatible, then $A$ and $B$ have a unique common fixed point.

Proof. Define the sequence $\left\{x_{n}\right\}$ and $\left\{z_{n}\right\}$ in $X$ defined by

$$
z_{n}=B x_{n+1}=A x_{n}
$$

If $z_{n}=z_{n+1}$, then $z_{n+1}$ is a point of coincidence of $A$ and $B$. Consequently, we can suppose that $z_{n} \neq z_{n+1}$ for all $n \in N$.

Now, by (6), we have

$$
\begin{aligned}
& d\left(A x_{n}, A x_{n+1}\right) \leq \phi\left(M\left(x_{n}, x_{n+1}\right)\right)+C \\
& \cdot \min \left\{d\left(B x_{n}, A x_{n}\right), d\left(B x_{n+1}, A x_{n+1}\right),\right. \\
& \left.d\left(B x_{n}, A x_{n+1}\right), d\left(B x_{n+1}, A x_{n}\right)\right\}=\phi\left(M\left(x_{n}, x_{n+1}\right)\right),
\end{aligned}
$$

where

$$
\begin{aligned}
& M\left(x_{n}, x_{n+1}\right)=\max \left\{d\left(B x_{n}, B x_{n+1}\right)\right. \\
& \frac{d\left(B x_{n}, A x_{n}\right)\left(d\left(B x_{n+1}, A x_{n+1}\right)+1\right)}{1+d\left(B x_{n}, B x_{n+1}\right)}, \\
& \left.\frac{d\left(B x_{n+1}, A x_{n+1}\right)\left(d\left(B x_{n}, A x_{n}\right)+1\right)}{1+d\left(B x_{n}, B x_{n+1}\right)}\right\} \\
& =\max \left\{d\left(z_{n-1}, z_{n}\right), \frac{d\left(z_{n-1}, z_{n}\right)\left(1+d\left(z_{n}, z_{n+1}\right)\right)}{1+d\left(z_{n-1}, z_{n}\right)},\right. \\
& \left.d\left(z_{n}, z_{n+1}\right)\right\}
\end{aligned}
$$

We consider the following cases:

(i) If $M\left(x_{n}, x_{n+1}\right)=d\left(z_{n-1}, z_{n}\right)$, from (9), we have

$$
d\left(z_{n}, z_{n+1}\right) \leq \phi\left(d\left(z_{n-1}, z_{n}\right)\right)<d\left(z_{n-1}, z_{n}\right) .
$$

(ii) If $M\left(x_{n}, x_{n+1}\right)=d\left(z_{n-1}, z_{n}\right)\left(1+d\left(z_{n}, z_{n+1}\right)\right) /(1+$ $\left.d\left(z_{n-1}, z_{n}\right)\right)$, from (9), we obtain

$$
\begin{aligned}
d\left(z_{n}, z_{n+1}\right) & \leq \phi\left(\frac{d\left(z_{n-1}, z_{n}\right)\left(1+d\left(z_{n}, z_{n+1}\right)\right)}{1+d\left(z_{n-1}, z_{n}\right)}\right) \\
& <\frac{d\left(z_{n-1}, z_{n}\right)\left(1+d\left(z_{n}, z_{n+1}\right)\right)}{1+d\left(z_{n-1}, z_{n}\right)} .
\end{aligned}
$$

Hence,

$$
d\left(z_{n}, z_{n+1}\right)<d\left(z_{n-1}, z_{n}\right) ;
$$

that is, (11) holds.

(iii) If $M\left(x_{n}, x_{n+1}\right)=d\left(z_{n}, z_{n+1}\right)$, from (9), we get

$$
d\left(z_{n}, z_{n+1}\right)<d\left(z_{n}, z_{n+1}\right),
$$

which is impossible.

In any case, we proved that (11) holds. Since $\left\{d\left(z_{n}, z_{n+1}\right)\right\}$ is decreasing sequence, it converges to a nonnegative number, $s \geq 0$. If $s>0$, then, letting $n \rightarrow+\infty$ in (9), we deduce

$$
s \leq \phi\left(\max \left\{s, \frac{s(1+s)}{1+s}, s\right\}\right)=\phi(s)<s
$$

which implies that $s=0$; that is,

$$
\lim _{n \rightarrow \infty} d\left(z_{n}, z_{n+1}\right)=0 .
$$

Suppose that $z_{n} \neq z_{m}$ for all $m \neq n$ and show that $\left\{z_{n}\right\}$ is GMS Cauchy sequence. First, we prove that the sequence $\left\{d\left(z_{n}, z_{n+2}\right)\right\}$ is bounded. Since $\lim _{n \rightarrow \infty} d\left(z_{n}, z_{n+1}\right)=0$, there exists $M>0$ such that $d\left(z_{n}, z_{n+1}\right) \leq M$ for all $n \in N$. If $d\left(z_{n}, z_{n+2}\right)>M$, for all $n \in N$, from (6), we have

$$
\begin{aligned}
& d\left(z_{n}, z_{n+2}\right)=d\left(A x_{n}, A x_{n+2}\right) \leq \phi\left(M\left(x_{n}, x_{n+2}\right)\right)+C \\
& \quad \cdot \min \left\{d\left(B x_{n}, A x_{n}\right), d\left(B x_{n+2}, A x_{n+2}\right),\right. \\
& \left.d\left(B x_{n}, A x_{n+2}\right), d\left(B x_{n+2}, A x_{n}\right)\right\}=\phi\left(M\left(x_{n}, x_{n+2}\right)\right) \\
& +C \min \left\{d\left(z_{n-1}, z_{n}\right), d\left(z_{n+1}, z_{n+2}\right), d\left(z_{n-1}, z_{n+2}\right),\right. \\
& \left.d\left(z_{n+1}, z_{n}\right)\right\}=\phi\left(M\left(x_{n}, x_{n+1}\right)\right) \text { as } n \longrightarrow \infty,
\end{aligned}
$$

where

$$
\begin{aligned}
& M\left(x_{n}, x_{n+2}\right)=\max \left\{d\left(B x_{n}, B x_{n+2}\right),\right. \\
& \frac{d\left(B x_{n}, A x_{n}\right)\left(d\left(B x_{n+2}, A x_{n+2}\right)+1\right)}{1+d\left(B x_{n}, B x_{n+2}\right)}, \\
& \left.\frac{d\left(B x_{n+2}, A x_{n+2}\right)\left(d\left(B x_{n}, A x_{n}\right)+1\right)}{1+d\left(B x_{n}, B x_{n+2}\right)}\right\} \\
& =\max \left\{d\left(z_{n-1}, z_{n+1}\right),\right. \\
& \frac{d\left(z_{n-1}, z_{n}\right)\left(1+d\left(z_{n+1}, z_{n+2}\right)\right)}{1+d\left(z_{n-1}, z_{n+1}\right)}, \\
& \left.\frac{d\left(z_{n+1}, z_{n+2}\right)\left(d\left(z_{n-1}, z_{n}\right)+1\right)}{1+d\left(z_{n-1}, z_{n+1}\right)}\right\}=d\left(z_{n-1}, z_{n+1}\right) .
\end{aligned}
$$

Hence,

$$
d\left(z_{n}, z_{n+2}\right) \leq \phi\left(d\left(z_{n-1}, z_{n+1}\right)\right)<d\left(z_{n-1}, z_{n+1}\right) .
$$


Thus the sequence $\left\{d\left(z_{n}, z_{n+2}\right)\right\}$ is decreasing and hence is bounded. Now, if

$$
\lim _{n \rightarrow \infty} d\left(z_{n}, z_{n+2}\right)=0
$$

dose not hold, then there exists a subsequence $\left\{z_{n_{k}}\right\}$ of $\left\{z_{n}\right\}$ such that $\lim _{n \rightarrow \infty} d\left(z_{n_{k}}, z_{n_{k}+2}\right)=s$. From

$$
\begin{aligned}
d\left(z_{n_{k}-1}, z_{n_{k}+1}\right) \leq & d\left(z_{n_{k}-1}, z_{n_{k}}\right)+d\left(z_{n_{k}}, z_{n_{k}+2}\right) \\
& +d\left(z_{n_{k}+1}, z_{n_{k}+2}\right), \\
d\left(z_{n_{k}}, z_{n_{k}+2}\right) \leq & d\left(z_{n_{k}-1}, z_{n_{k}}\right)+d\left(z_{n_{k}-1}, z_{n_{k}+1}\right) \\
& +d\left(z_{n_{k}+1}, z_{n_{k}+2}\right),
\end{aligned}
$$

we obtain that

$$
\lim _{k \rightarrow+\infty} d\left(z_{n_{k}-1}, z_{n_{k}+1}\right)=s
$$

Now, by (6), with $x=x_{n_{k}}$ and $y=x_{n_{k}+2}$, we have

$$
d\left(z_{n_{k}}, z_{n_{k}+2}\right) \leq \phi\left(d\left(z_{n_{k}-1}, z_{n_{k}+1}\right)\right),
$$

which tends to $s \leq \phi(s)$ as $k \rightarrow \infty$; hence, $s=0$.

Now, if possible, let $\left\{z_{n}\right\}$ be not a Cauchy sequence. Then there exists $\epsilon>0$ such that, for $k>0$, there exist $n_{k}>m_{k} \geq k$ for which we can find subsequences $\left\{z_{n_{k}}\right\}$ and $\left\{z_{m_{k}}\right\}$ of $\left\{z_{n}\right\}$ such that $n(k)$ is the smallest index for which

$$
\begin{gathered}
d\left(z_{n_{k}}, z_{m_{k}}\right) \geq \epsilon, \\
d\left(z_{n_{k}-1}, z_{m_{k}}\right)<\epsilon .
\end{gathered}
$$

Now, using (24) and the rectangular inequality, we get

$$
\begin{aligned}
\epsilon & \leq d\left(z_{n_{k}}, z_{m_{k}}\right) \\
& \leq d\left(z_{n_{k}}, z_{n_{k}-2}\right)+d\left(z_{n_{k}-2}, z_{n_{k}-1}\right)+d\left(z_{n_{k}-1}, z_{m_{k}}\right) \\
& <d\left(z_{n_{k}}, z_{n_{k}-2}\right)+d\left(z_{n_{k}-2}, z_{n_{k}-1}\right)+\epsilon .
\end{aligned}
$$

Letting $k \rightarrow+\infty$ in the above inequality, using (16) and (20), we obtain

$$
\lim _{k \rightarrow+\infty} d\left(z_{n_{k}}, z_{m_{k}}\right)=\epsilon^{+}
$$

From

$$
\begin{aligned}
& d\left(z_{n_{k}}, z_{m_{k}}\right)-d\left(z_{m_{k}}, z_{m_{k}-1}\right)-d\left(z_{n_{k}-1}, z_{n_{k}}\right) \\
& \quad \leq d\left(z_{n_{k}-1}, z_{m_{k}-1}\right) \\
& \quad \leq d\left(z_{n_{k}-1}, z_{n_{k}}\right)+d\left(z_{m_{k}}, z_{n_{k}}\right)+d\left(z_{m_{k}-1}, z_{m_{k}}\right),
\end{aligned}
$$

letting $k \rightarrow+\infty$, we obtain

$$
\lim _{k \rightarrow \infty} d\left(z_{n_{k}-1}, z_{m_{k}-1}\right)=\epsilon
$$

From (6), with $x=x_{n_{k}}$ and $y=x_{m_{k}}$, we get

$$
\begin{aligned}
& d\left(A x_{m_{k}}, A x_{n_{k}}\right) \leq \phi\left(M\left(x_{m_{k}}, x_{n_{k}}\right)\right)+C \\
& \cdot \min \left\{d\left(B x_{n_{k}}, A x_{n_{k}}\right), d\left(B x_{m_{k}}, A x_{m_{k}}\right),\right. \\
& \left.d\left(B x_{n_{k}}, A x_{m_{k}}\right), d\left(B x_{m_{k}}, A x_{n_{k}}\right)\right\}=\phi\left(M \left(x_{m_{k}},\right.\right. \\
& \left.\left.x_{n_{k}}\right)\right)+C \min \left\{d\left(z_{n_{k}-1}, z_{n_{k}}\right), d\left(z_{m_{k}-1}, z_{m_{k}}\right),\right. \\
& \left.d\left(z_{n_{k}-1}, z_{m_{k}}\right), d\left(z_{m_{k}-1}, z_{n_{k}}\right)\right\},
\end{aligned}
$$

where

$$
\begin{aligned}
& M\left(x_{m_{k}}, x_{n_{k}}\right)=\max \left\{d\left(B x_{m_{k}}, B x_{n_{k}}\right),\right. \\
& \frac{d\left(B x_{m_{k}}, A x_{m_{k}}\right)\left(d\left(B x_{n_{k}}, A x_{n_{k}}\right)+1\right)}{1+d\left(B x_{m_{k}}, B x_{n_{k}}\right)}, \\
& \left.\frac{d\left(B x_{n_{k}}, A x_{n_{k}}\right)\left(d\left(B x_{m_{k}}, A x_{m_{k}}\right)+1\right)}{1+d\left(B x_{m_{k}}, B x_{n_{k}}\right)}\right\} \\
& =\max \left\{d\left(z_{m_{k}-1}, z_{n_{k}-1}\right),\right. \\
& \frac{d\left(z_{m_{k}-1}, z_{m_{k}}\right)\left(d\left(z_{n_{k}-1}, z_{n_{k}}\right)+1\right)}{1+d\left(z_{m_{k}-1}, z_{n_{k}-1}\right)}, \\
& \left.\frac{d\left(z_{n_{k}-1}, z_{n_{k}}\right)\left(d\left(z_{m_{k}-1}, z_{m_{k}}\right)+1\right)}{1+d\left(z_{m_{k}-1}, z_{n_{k}-1}\right)}\right\}
\end{aligned}
$$

Now, using the continuity of $\phi$ as $k \rightarrow+\infty$, we obtain

$$
\epsilon \leq \phi(\epsilon)+0<\epsilon,
$$

which implies that $\epsilon=0$, a contradiction with $\epsilon>0$. Hence, $\left\{z_{n}\right\}$ is a GMS Cauchy sequence. Since $(B X, d)$ is complete GMS, there exists $z \in B X$ such that $\lim _{n \rightarrow \infty} z_{n}=z$. Let $u \in X$ be such that $B u=z$, applying (6) with $x=x_{n_{k}}$ :

$$
\begin{aligned}
& d\left(A u, A x_{n_{k}}\right) \leq \phi\left(M\left(u, x_{n_{k}}\right)\right)+L \\
& \quad \min \left\{d\left(B x_{n_{k}}, A x_{n_{k}}\right), d(B u, A u), d\left(B x_{n_{k}}, A u\right),\right. \\
& \left.d\left(B u, A x_{n_{k}}\right)\right\}=\phi\left(M\left(u, x_{n_{k}}\right)\right)+L \\
& \quad \cdot \min \left\{d\left(z_{n_{k}-1}, z_{n_{k}}\right), d(B u, A u), d\left(z_{n_{k}-1}, A u\right)\right. \\
& \left.d\left(z, z_{n_{k}}\right)\right\}=\phi\left(M\left(u, x_{n_{k}}\right)\right)+0
\end{aligned}
$$


where

$$
\begin{aligned}
& M\left(u, x_{n_{k}}\right)=\max \left\{d\left(B u, B x_{n_{k}}\right),\right. \\
& \frac{d(B u, A u)\left(d\left(B x_{n_{k}}, A x_{n_{k}}\right)+1\right)}{1+d\left(B u, B x_{n_{k}}\right)}, \\
& \left.\frac{d\left(B x_{n_{k}}, A x_{n_{k}}\right)(d(B u, A u)+1)}{1+d\left(B u, B x_{n_{k}}\right)}\right\} \\
& =\max \left\{d\left(z, z_{n_{k}-1}\right),\right. \\
& \frac{d(B u, A u)\left(d\left(z_{n_{k}-1}, z_{n_{k}}\right)+1\right)}{1+d\left(B u, z_{n_{k}-1}\right)}, \\
& \left.\frac{d\left(z_{n_{k}-1}, z_{n_{k}}\right)(d(B u, A u)+1)}{1+d\left(B u, z_{n_{k}-1}\right)}\right\}=d(B u, A u)
\end{aligned}
$$

We get from (32) that

$$
\begin{aligned}
& d(B u, A u) \leq \liminf _{k \rightarrow \infty}\left[d\left(B u, z_{n_{k}-1}\right)+d\left(z_{n_{k}-1}, z_{n_{k}}\right)\right. \\
& \left.\quad+d\left(A u, A x_{n_{k}}\right)\right] \leq \liminf _{k \rightarrow \infty} d\left(A u, A x_{n_{k}}\right) \\
& \quad=\phi(d(B u, A u))<d(B u, A u),
\end{aligned}
$$

which implies that $d(B u, A u)=0$; that is, $z=B u=A u$ and so $z$ is a point of coincidence for $A$ and $B$.

Now, we prove that $z$ is the unique point of coincidence of $A$ and $B$. Let $x$ and $y$ be arbitrary points of coincidence of $A$ and $B$ such that $x=A u=B u$ and $y=A v=B v$. Using condition (6), it follows that

$$
\begin{aligned}
& d(x, y)=d(A u, A v) \leq \phi(\max \{d(B u, B v) \\
& \quad \frac{d(B u, A u)(d(B v, A v)+1)}{1+d(B u, B v)}, \\
& \left.\left.\quad \frac{d(B v, A v)(d(B u, A u)+1)}{1+d(B u, B v)}\right\}\right)+C \min \{d(B v, A v), \\
& d(B u, A u), d(B v, A u), d(B u, A v)\}=\phi(d(B u, B v)) \\
& <d(B u, B v)=d(x, y),
\end{aligned}
$$

which implies that $d(x, y)=0$. Thus, $x=y$ and $A, B$ have a unique point of coincidence.

Next, we prove that $z=A z=B z$. If $z$ is a point of coincidence of $A$ and $B$ as $A$ and $B$ are weakly compatible, we obtain that $A z=A A u=A B u=B A u=B z$ and so $z=A z=B z$. Consequently, $z$ is unique common fixed point of $A$ and $B$.
Example 11. Suppose $(X, d)$ as in Example 2; let $A, B: \mathrm{X} \rightarrow X$ and $\phi(t):[0, \infty) \rightarrow[0, \infty)$, defined by $A x=(1 / 2) x, B x=x$, and $\phi(t)=(t / 2), \forall t \in[0, \infty)$.

Then $A, B$ and $\phi$ satisfy all the conditions of Theorem 10 . Hence, 0 is unique coincidence and common fixed point of $A$ and $B$.

Corollary 12. Replace condition (6) in Theorem 10 with the following condition:

$$
\begin{aligned}
& d(A x, A y) \leq a_{1} d(B x, B y) \\
& +a_{2} \frac{d(B x, A x)(d(B y, A y)+1)}{1+d(B x, B y)} \\
& +a_{3} \frac{d(B y, A y)(d(B x, A x)+1)}{1+d(B x, B y)}+L \\
& \cdot \min \{d(B x, A x), d(B y, A y), d(B x, A y), \\
& d(B y, A x)\},
\end{aligned}
$$

where $a_{1}, a_{2}, a_{3}, L \geq 0$ and $a_{1}+a_{2}+a_{3}<1$.

Then $A$ and $B$ have a unique point of coincidence in $X$. Moreover, if $A$ and $B$ are weakly compatible, then $A$ and $B$ have a unique common fixed point.

Corollary 13. Put B =I (the identity mapping) in Theorem 10. Then one can get a unique fixed point of $A$.

Remark 14 (see [5, Theorem 7]). It is spatial case of Theorem 10. Next, we introduce some coincidence point theorems for two $(\alpha, \psi, \phi)$-contractions self-mappings of rational type in complete GMS.

Theorem 15. Let $(X, d)$ be a GMS and let $A, B: X \rightarrow X$ be two self-mappings satisfying the following conditions:

$$
\begin{aligned}
& \phi(\beta(B x, B y) d(A x, A y)) \\
& \quad \leq \phi(M(x, y))-\psi(M(x, y)) \quad \forall x, y \in X,
\end{aligned}
$$

where $M(x, y)$ is as in Theorem 10; $A X \subset B X$, and $(B X, d)$ is a complete GMS.

Consider also that the next conditions hold:

(i) $\exists x_{0} \in X$ such that $\beta\left(A x_{0}, B x_{0}\right) \geq 1$.

(ii) $A$ is $B-\beta$-admissible.

(iii) $X$ is $\beta$-regular and $\beta\left(x_{m}, x_{n}\right) \geq 1$, for each $x_{n} \in X$, and $\forall m, n \in N, m \neq n$.

(iv) Either $\beta(B x, B y) \geq 1$ or $\beta(B y, B x) \geq 1$ whenever $B x=$ $A x$ and $B y=A y$.

(v) $\psi:[0,+\infty) \rightarrow[0,+\infty)$ is a lower semicontinuous function and $\psi(t)=0 \Leftrightarrow t=0$.

Then $A$ and $B$ have a unique point of coincidence in $X$. Moreover, if $A$ and $B$ are weakly compatible, then $A$ and $B$ have a unique common fixed point. 
Proof. Suppose that $x_{0} \in X, \beta\left(B x_{0}, A x_{0}\right) \geq 1$. Define $\left\{z_{n}\right\}$ and $\left\{x_{n}\right\}$ as two sequences in $X$ such that $z_{n}=B x_{n+1}=$ $A x_{n}, n=0,1,2,3, \ldots$. If $z_{n}=z_{n+1}$, then $B x_{n+1}=A x_{n+1}$, which implies that $x_{n+1}$ is a coincidence point of $A$ and $B$. Consequently, we can suppose that $z_{n} \neq z_{n+1}$ for all $n \in$ $N$. From (i), we get that $\beta\left(B x_{0}, A x_{0}\right)=\beta\left(B x_{0}, B x_{1}\right) \geq 1$. Also, by (ii), we have that $\beta\left(A x_{0}, A x_{1}\right)=\beta\left(B x_{1}, B x_{2}\right) \geq$ 1, $\beta\left(A x_{1}, A x_{2}\right)=\beta\left(B x_{2}, B x_{3}\right) \geq 1$. Continuous with this process, we obtain that $\beta\left(B x_{n}, B x_{n+1}\right) \geq 1$. Now, by using (37), we get

$$
\begin{aligned}
& \phi\left(d\left(\mathrm{~A} x_{n}, A x_{n+1}\right)\right) \\
& \quad \leq \phi\left(\beta\left(B x_{n}, B x_{n+1}\right) d\left(A x_{n}, A x_{n+1}\right)\right) \\
& \quad \leq \phi\left(M\left(x_{n}, x_{n+1}\right)\right)-\psi\left(M\left(x_{n}, x_{n+1}\right)\right),
\end{aligned}
$$

where

$$
\begin{aligned}
& M\left(x_{n}, x_{n+1}\right)=\max \left\{d\left(B x_{n}, B x_{n+1}\right),\right. \\
& \frac{d\left(B x_{n}, A x_{n}\right)\left(d\left(B x_{n+1}, A x_{n+1}\right)+1\right)}{1+d\left(B x_{n}, B x_{n+1}\right)}, \\
& \left.\frac{d\left(B x_{n+1}, A x_{n+1}\right)\left(d\left(B x_{n}, A x_{n}\right)+1\right)}{1+d\left(B x_{n}, B x_{n+1}\right)}\right\} \\
& =\max \left\{d\left(z_{n-1}, z_{n}\right), \frac{d\left(z_{n-1}, z_{n}\right)\left(1+d\left(z_{n}, z_{n+1}\right)\right)}{1+d\left(z_{n-1}, z_{n}\right)},\right. \\
& \left.d\left(z_{n}, z_{n+1}\right)\right\} .
\end{aligned}
$$

We consider the following cases:

(i) If $M\left(x_{n}, x_{n+1}\right)=d\left(z_{n-1}, z_{n}\right)$, from (38), we have

$$
\begin{aligned}
\phi\left(d\left(z_{n}, z_{n+1}\right)\right) & \leq \phi\left(d\left(z_{n-1}, z_{n}\right)\right)-\psi\left(d\left(z_{n-1}, z_{n}\right)\right) \\
& <\phi\left(d\left(z_{n-1}, z_{n}\right)\right) .
\end{aligned}
$$

Since $\phi$ is nondecreasing, we have

$$
d\left(z_{n}, z_{n+1}\right)<\phi\left(d\left(z_{n-1}, z_{n}\right)\right) .
$$

(ii) If $M\left(x_{n}, x_{n+1}\right)=d\left(z_{n-1}, z_{n}\right)\left(1+d\left(z_{n}, z_{n+1}\right)\right) /(1+$ $\left.d\left(z_{n-1}, z_{n}\right)\right)$, from (38), we obtain

$$
\begin{aligned}
\phi( & \left.d\left(z_{n}, z_{n+1}\right)\right) \\
\leq & \phi\left(\frac{d\left(z_{n-1}, z_{n}\right)\left(1+d\left(z_{n}, z_{n+1}\right)\right)}{1+d\left(z_{n-1}, z_{n}\right)}\right) \\
& -\psi\left(\frac{d\left(z_{n-1}, z_{n}\right)\left(1+d\left(z_{n}, z_{n+1}\right)\right)}{1+d\left(z_{n-1}, z_{n}\right)}\right) \\
< & \phi\left(\frac{d\left(z_{n-1}, z_{n}\right)\left(1+d\left(z_{n}, z_{n+1}\right)\right)}{1+d\left(z_{n-1}, z_{n}\right)}\right) .
\end{aligned}
$$

The nondecreasing property of $\phi$ implies that

$$
\begin{aligned}
d\left(z_{n}, z_{n+1}\right) & <\frac{d\left(z_{n-1}, z_{n}\right)\left(1+d\left(z_{n}, z_{n+1}\right)\right)}{1+d\left(z_{n-1}, z_{n}\right)} \\
& \Longrightarrow d\left(z_{n}, z_{n+1}\right)+d\left(z_{n}, z_{n+1}\right) d\left(z_{n-1}, z_{n}\right) \\
& <d\left(z_{n-1}, z_{n}\right)+d\left(z_{n}, z_{n+1}\right) d\left(z_{n-1}, z_{n}\right) \\
& \Longrightarrow d\left(z_{n}, z_{n+1}\right)<d\left(z_{n-1}, z_{n}\right) .
\end{aligned}
$$

Hence, (41) is obtained.

(iii) If $\left.M\left(x_{n}, x_{n+1}\right)=d\left(z_{n}, z_{n+1}\right)\right)$, by (38), we obtain

$$
\begin{aligned}
\phi\left(d\left(z_{n}, z_{n+1}\right)\right) & \leq \phi\left(d\left(z_{n}, z_{n+1}\right)\right)-\psi\left(d\left(z_{n}, z_{n+1}\right)\right) \\
& <\phi\left(d\left(z_{n}, z_{n+1}\right)\right) ;
\end{aligned}
$$

this is a contradiction.

In any case, we proved that (41) holds. Since $\left\{d\left(z_{n}, z_{n+1}\right)\right\}$ is decreasing, it converges to a nonnegative number, $s \geq 0$. If $s>0$, then, letting $n \rightarrow+\infty$ in (37), we deduce

$$
s \leq \phi(s)<s,
$$

which implies that $s=0$; that is,

$$
\lim _{n \rightarrow \infty} d\left(z_{n}, z_{n+1}\right)=0 \text {. }
$$

Suppose that $z_{n} \neq z_{m}$ for all $m \neq n$ and prove that $\left\{z_{n}\right\}$ is GMS Cauchy sequence. First, we show that the sequence $\left\{d\left(z_{n}, z_{n+2}\right)\right\}$ is bounded. Since $\lim _{n \rightarrow \infty} d\left(z_{n}, z_{n+1}\right)=0$, there exists $L>0$ such that $d\left(z_{n}, z_{n+1}\right) \leq L$ for all $n \in N$. If $d\left(z_{n}, z_{n+2}\right)>L$, for all $n \in N$, from (37), we have

$$
\begin{aligned}
& \phi\left(d\left(z_{n}, z_{n+2}\right)\right) \\
& \quad \leq \phi\left(\beta\left(d\left(B x_{n}, B x_{n+2}\right)\right) d\left(A x_{n}, A x_{n+2}\right)\right) \\
& \quad=d\left(A x_{n}, A x_{n+2}\right) \\
& \quad \leq \phi\left(M\left(x_{n}, x_{n+2}\right)\right)-\psi\left(M\left(x_{n}, x_{n+2}\right)\right) \\
& \quad<\phi\left(M\left(x_{n}, x_{n+1}\right)\right)=\phi\left(d\left(z_{n-1}, z_{n+1}\right)\right)
\end{aligned}
$$

where

$$
\begin{aligned}
& M\left(x_{n}, x_{n+2}\right)=\max \left\{d\left(B x_{n}, B x_{n+2}\right),\right. \\
& \frac{d\left(B x_{n}, A x_{n}\right)\left(d\left(B x_{n+2}, A x_{n+2}\right)+1\right)}{1+d\left(B x_{n}, B x_{n+2}\right)}, \\
& \left.\frac{d\left(B x_{n+2}, A x_{n+2}\right)\left(d\left(B x_{n}, A x_{n}\right)+1\right)}{1+d\left(B x_{n}, B x_{n+2}\right)}\right\} \\
& =\max \left\{d\left(z_{n-1}, z_{n+1}\right),\right.
\end{aligned}
$$




$$
\begin{aligned}
& \frac{d\left(z_{n-1}, z_{n}\right)\left(1+d\left(z_{n+1}, z_{n+2}\right)\right)}{1+d\left(z_{n-1}, z_{n+1}\right)}, \\
& \begin{aligned}
\left.\frac{d\left(z_{n+1}, z_{n+2}\right)\left(d\left(z_{n-1}, z_{n}\right)+1\right)}{1+d\left(z_{n-1}, z_{n+1}\right)}\right\}=d\left(z_{n-1}, z_{n+1}\right) \\
\text { as } n \longrightarrow \infty .
\end{aligned}
\end{aligned}
$$

Hence,

$$
d\left(z_{n}, z_{n+2}\right) \leq \phi\left(d\left(z_{n-1}, z_{n+1}\right)\right)<d\left(z_{n-1}, z_{n+1}\right) .
$$

Thus the sequence $\left\{d\left(z_{n}, z_{n+2}\right)\right\}$ is decreasing and hence is bounded. If, for some $n \in N$, we have $d\left(z_{n-1}, z_{n+1}\right) \leq L$ and $d\left(z_{n}, z_{n+2}\right)>L$, then, from (49), we get

$$
d\left(z_{n}, z_{n+2}\right)<L
$$

which is a contradiction. Then $\left\{d\left(z_{n}, z_{n+2}\right)\right\}$ is bounded. Now, if

$$
\lim _{n \rightarrow \infty} d\left(z_{n}, z_{n+2}\right)=0
$$

dose not hold, then there exists a subsequence $\left\{z_{n_{k}}\right\}$ of $\left\{z_{n}\right\}$ such that $\lim _{n \rightarrow \infty} d\left(z_{n_{k}}, z_{n_{k}+2}\right)=s$. From

$$
\begin{aligned}
d\left(z_{n_{k}-1}, z_{n_{k}+1}\right) \leq & d\left(z_{n_{k}-1}, z_{n_{k}}\right)+d\left(z_{n_{k}}, z_{n_{k}+2}\right) \\
& +d\left(z_{n_{k}+1}, z_{n_{k}+2}\right) \\
d\left(z_{n_{k}}, z_{n_{k}+2}\right) \leq & d\left(z_{n_{k}-1}, z_{n_{k}}\right)+d\left(z_{n_{k}-1}, z_{n_{k}+1}\right) \\
& +d\left(z_{n_{k}+1}, z_{n_{k}+2}\right)
\end{aligned}
$$

we obtain that

$$
\lim _{k \rightarrow+\infty} d\left(z_{n_{k}-1}, z_{n_{k}+1}\right)=s
$$

Now, by (37), one can obtain that

$$
\begin{aligned}
\phi\left(d\left(z_{n_{k}}, z_{n_{k}+2}\right)\right) \leq & \phi\left(d\left(z_{n_{k}-1}, z_{n_{k}+1}\right)\right) \\
& -\psi\left(d\left(z_{n_{k}-1}, z_{n_{k}+1}\right)\right) \\
& \Longrightarrow \phi(s)<\phi(s)
\end{aligned}
$$

$$
\text { as } n \longrightarrow \infty \text {, }
$$

which implies that $s=0$.

Now, if possible, let $\left\{z_{n}\right\}$ be not a Cauchy sequence. Then there exists $\epsilon>0$ for which we can find subsequences $\left\{z_{n_{k}}\right\}$ and $\left\{z_{m_{k}}\right\}$ of $\left\{z_{n}\right\}$ with $n_{k}>m_{k} \geq k$ such that

$$
d\left(z_{n_{k}}, z_{m_{k}}\right) \geq \epsilon
$$

Further, corresponding to $m_{k}$, we can choose $n_{k}$ in such a way that it is the smallest integer for which

$$
d\left(z_{n_{k}-1}, z_{m_{k}}\right)<\epsilon
$$

Now, using (55) and (56) and the rectangular inequality, we get

$$
\begin{aligned}
\epsilon & \leq d\left(z_{n_{k}}, z_{m_{k}}\right) \\
& \leq d\left(z_{n_{k}}, z_{n_{k}-2}\right)+d\left(z_{n_{k}-2}, z_{n_{k}-1}\right)+d\left(z_{n_{k}-1}, z_{m_{k}}\right) \\
& <d\left(z_{n_{k}}, z_{n_{k}-2}\right)+d\left(z_{n_{k}-2}, z_{n_{k}-1}\right)+\epsilon .
\end{aligned}
$$

Letting $k \rightarrow+\infty$ in the above inequality, using (46) and (51), we obtain

$$
\lim _{k \rightarrow \infty} d\left(z_{n_{k}}, z_{m_{k}}\right)=\epsilon^{+}
$$

From

$$
\begin{aligned}
& d\left(z_{n_{k}}, z_{m_{k}}\right)-d\left(z_{m_{k}}, z_{m_{k}-1}\right)-d\left(z_{n_{k}-1}, z_{n_{k}}\right) \\
& \quad \leq d\left(z_{n_{k}-1}, z_{m_{k}-1}\right) \\
& \quad \leq d\left(z_{n_{k}-1}, z_{n_{k}}\right)+d\left(z_{m_{k}}, z_{n_{k}}\right)+d\left(z_{m_{k}-1}, z_{m_{k}}\right),
\end{aligned}
$$

letting $k \rightarrow+\infty$, we obtain

$$
\lim _{k \rightarrow \infty} d\left(z_{n_{k}-1}, z_{m_{k}-1}\right)=\epsilon
$$

From (37), with $x=x_{n_{k}}$ and $y=x_{m_{k}}$, we get

$$
\begin{aligned}
& \phi\left(d\left(A x_{m_{k}}, A x_{n_{k}}\right)\right) \\
& \leq \phi\left(\beta\left(d\left(B x_{m_{k}}, B x_{n_{k}}\right)\right) d\left(A x_{m_{k}}, A x_{n_{k}}\right)\right) \\
& \leq \phi\left(M\left(x_{m_{k}}, x_{n_{k}}\right)\right)-\psi\left(M\left(x_{m_{k}}, x_{n_{k}}\right)\right),
\end{aligned}
$$

where

$$
\begin{aligned}
& M\left(x_{m_{k}}, x_{n_{k}}\right)=\max \left\{d\left(B x_{m_{k}}, B x_{n_{k}}\right),\right. \\
& \frac{d\left(B x_{m_{k}}, A x_{m_{k}}\right)\left(d\left(B x_{n_{k}}, A x_{n_{k}}\right)+1\right)}{1+d\left(B x_{m_{k}}, B x_{n_{k}}\right)}, \\
& \left.\frac{d\left(B x_{n_{k}}, A x_{n_{k}}\right)\left(d\left(B x_{m_{k}}, A x_{m_{k}}\right)+1\right)}{1+d\left(B x_{m_{k}}, B x_{n_{k}}\right)}\right\} \\
& =\max \left\{d\left(z_{m_{k}-1}, z_{n_{k}-1}\right),\right. \\
& \frac{d\left(z_{m_{k}-1}, z_{m_{k}}\right)\left(d\left(z_{n_{k}-1}, z_{n_{k}}\right)+1\right)}{1+d\left(z_{m_{k}-1}, z_{n_{k}-1}\right)}, \\
& \left.\frac{d\left(z_{n_{k}-1}, z_{n_{k}}\right)\left(d\left(z_{m_{k}-1}, z_{m_{k}}\right)+1\right)}{1+d\left(z_{m_{k}-1}, z_{n_{k}-1}\right)}\right\} .
\end{aligned}
$$

Now, using the continuity of $\phi$ as $k \rightarrow+\infty$, we obtain

$$
\epsilon \leq \phi(\epsilon)<\epsilon \text {. }
$$


A contradiction is obtained with $\epsilon>0$, and then $\epsilon=0$, and hence $\left\{z_{n}\right\}$ is a GMS Cauchy sequence. Since $(B X, d)$ is complete GMS, there exists $z \in B X$ such that $\lim _{n \rightarrow \infty} z_{n}=z$. Let $w \in X$ be such that $B u=z$, applying (37) with $x=x_{n_{k}}$ :

$$
\begin{aligned}
\phi\left(d\left(A u, A x_{n_{k}}\right)\right) \leq & \phi\left(M\left(u, x_{n_{k}}\right)\right) \\
& -\phi\left(M\left(u, x_{n_{k}}\right)\right),
\end{aligned}
$$

where

$$
\begin{aligned}
& M\left(u, x_{n_{k}}\right)=\max \left\{d\left(B u, B x_{n_{k}}\right),\right. \\
& \frac{d(B u, A u)\left(d\left(B x_{n_{k}}, A x_{n_{k}}\right)+1\right)}{1+d\left(B u, B x_{n_{k}}\right)}, \\
& \left.\frac{d\left(B x_{n_{k}}, A x_{n_{k}}\right)(d(B u, A u)+1)}{1+d\left(B u, B x_{n_{k}}\right)}\right\} \\
& =\max \left\{d\left(z, z_{n_{k}-1}\right),\right. \\
& \frac{d(B u, A u)\left(d\left(z_{n_{k}-1}, z_{n_{k}}\right)+1\right)}{1+d\left(B u, z_{n_{k}-1}\right)}, \\
& \left.\frac{d\left(z_{n_{k}-1}, z_{n_{k}}\right)(d(B u, A u)+1)}{1+d\left(B u, z_{n_{k}-1}\right)}\right\}=d(B u, A u)
\end{aligned}
$$

We get from (64) that

$$
\begin{aligned}
& d(B u, A u) \leq \liminf _{k \rightarrow \infty}\left[d\left(B u, z_{n_{k}-1}\right)+d\left(z_{n_{k}-1}, z_{n_{k}}\right)\right. \\
& \left.+d\left(A u, A x_{n_{k}}\right)\right] \leq \liminf _{k \rightarrow \infty} d\left(A u, A x_{n_{k}}\right) \\
& \quad=\phi(d(B u, A u))<d(B u, A u),
\end{aligned}
$$

which implies that $d(B u, A u)=0$; that is, $z=B u=A u$ and so $z$ is a coincidence point for $A$ and $B$.

Now, we prove that $z$ is the unique coincidence point of $A$ and $B$. Let $x$ and $y$ be arbitrary coincidence points of $A$ and $B$ such that $x=A u=B u$ and $y=A v=B v$. Using condition (37), it follows that

$$
\begin{aligned}
& \phi(d(x, y))=\phi(d(A u, A v)) \leq \phi(\max \{d(B u, B v), \\
& \frac{d(B u, A u)(d(B v, A v)+1)}{1+d(B u, B v)}, \\
& \left.\left.\frac{d(B v, A v)(d(B u, A u)+1)}{1+d(B u, B v)}\right\}\right) \\
& -\psi(\max \{d(B u, B v),
\end{aligned}
$$

$$
\begin{aligned}
& \frac{d(B u, A u)(d(B v, A v)+1)}{1+d(B u, B v)}, \\
& \left.\left.\frac{d(B v, A v)(d(B u, A u)+1)}{1+d(B u, B v)}\right\}\right)=\phi(d(B u, B v)) \\
& -\psi(d(B u, B v))<\phi(d(B u, B v))=\phi(d(x, y)),
\end{aligned}
$$

which implies that $d(x, y)=0$. Thus, $x=y$ and $A, B$ have a unique coincidence point.

As in the conclusion in the last paragraph of the proof of Theorem 10 and the weakly compatible property of $A$ and $B$, we obtain that $A$ and $B$ have unique common fixed point.

Corollary 16. Put $B=I$ in Theorem 15 . Then one can get a unique fixed point of $A$.

Remark 17 (see [5, Theorem 16]). It is spatial case of Theorem 15 .

\section{An Application in Dynamical Programming}

The aim of this section is to use Theorem 10 to study the existence and uniqueness of solutions of the following system of functional equations:

$$
\begin{aligned}
& w(x)=\sup _{b \in E}\{h(a, b)+F(a, b, z(G(a, b)))\}, \\
& z(x)=\sup _{b \in E}\{h(a, b)+F(a, b, w(G(a, b)))\},
\end{aligned}
$$

which are used in dynamic programming (see [20-22]), where $E$ is a state space, $S$ is a decision space, and $x \in S, y \in E$, $h: S \times E \rightarrow \mathfrak{R}, G: S \times E \rightarrow S$, and $F: S \times E \times \mathfrak{R} \rightarrow \mathfrak{R}$ are considered operators.

We denote by $B(S)$ the set of all bounded functionals on $S$. Also, we define $\|\cdot\|_{\infty}$ by

$$
\|v\|_{\infty}=\sup _{x \in S}|v(x)|, \quad \forall v \in B(S) .
$$

Remark 18. We note that the space $\left(B(S),\|\cdot\|_{\infty}\right)$ is Banach, where the distance function in $B(S)$ is defined as follows:

$$
d_{\infty}\left(T_{1}, T_{2}\right)=\sup _{x \in S}\left|T_{1}(x)-T_{2}(x)\right| \quad \forall T_{1}, T_{2} \in B(S) .
$$

Lemma 19 (see [5]). Let $F_{1}, F_{2}: S \rightarrow \mathfrak{R}$ be bounded functionals; then

$$
\left|\sup _{x \in S} F_{1}(x)-\sup _{x \in S} F_{2}(x)\right| \leq \sup _{x \in S}\left|F_{1}(x)-F_{1}(x)\right| .
$$

Proposition 20. Suppose that $h, F(\cdot, \cdot, 0), F(\cdot, \cdot, 1): S \times E \rightarrow \Re$ are three bounded functionals and

$$
\begin{aligned}
\left|F\left(a, b, t_{1}\right)-F\left(a, b, t_{2}\right)\right| & \leq C\left|t_{1}-t_{2}\right|, \\
C & \geq 0
\end{aligned}
$$


Also, let $O: B(S) \rightarrow B(S)$ be an operator defined as follows:

$$
\begin{aligned}
& (O w)(b)=\sup _{b \in E}\{h(a, b)+F(a, b, z(G(a, b)))\}, \\
& \forall a \in S, \\
& z(a)=\sup _{b \in E}\{h(a, b)+F(a, b, w(G(a, b)))\},
\end{aligned}
$$

$\forall a \in S$

For all $w \in B(S)$ and $x \in S, O$ is well defined.

Proof. It is enough to prove that $O: B(S) \rightarrow B(S)$ is bounded for all $w \in B(S)$. From the boundedness of $w, h$, and $F$, we have

$$
\begin{aligned}
\mid(O w) & (x)\left|\leq \sup _{b \in E}\right| h(a, b)+F(a, b, z(G(a, b))) \mid \\
\leq & \sup _{b \in E}|h(a, b)| \\
& +\sup _{b \in E}|F(a, b, z(G(a, b)))-F(a, b, 0)| \\
& +\sup _{b \in E}|F(a, b, 0)-F(a, b, 1)|+\sup _{b \in E}|F(a, b, 1)| \\
\leq & \sup _{b \in E}|h(a, b)|+\sup _{b \in E}|z(G(a, b))|+1 \\
& +\sup _{b \in E}|F(a, b, 1)| \\
\leq & \sup _{b \in E}|h(a, b)| \\
& +\sup _{b \in E}\left|\sup _{b \in E}\{h(a, b)+F(a, b, w(G(a, b)))\}\right|+1 \\
& +\sup _{b \in E}|F(a, b, 1)| \\
\leq & 2 \sup _{b \in E}|h(a, b)|+\sup _{b \in E}|w(G(a, b))|+2 \\
& +2 \sup _{b \in E}|F(a, b, 1)| \leq C^{\prime}, \quad C^{\prime}>0,
\end{aligned}
$$

which give the boundedness of $O(w)$ on $w$. Hence, $R$ is well defined.

Theorem 21. Consider the assumptions of Proposition 20 and the following property:

$$
\begin{aligned}
& d\left(F\left(a, b, z\left(w_{1}(G(a, b))\right)\right), F\left(a, b, z\left(w_{2}(G(a, b))\right)\right)\right) \\
& \quad \leq \phi\left(M\left(w_{1}, w_{2}\right)\right)+C m\left(w_{1}, w_{2}\right),
\end{aligned}
$$

where

$$
\begin{aligned}
& M\left(w_{1}, w_{2}\right)=\max \left\{d_{\infty}\left(z w_{1}, z w_{2}\right),\right. \\
& \frac{d_{\infty}\left(z w_{1}, O w_{1}\right)\left(d_{\infty}\left(z w_{2}, O w_{2}\right)+1\right)}{1+d_{\infty}\left(z w_{1}, z w_{2}\right)}, \\
& \left.\frac{d_{\infty}\left(z w_{2}, O w_{2}\right)\left(d_{\infty}\left(z w_{1}, O x\right)+1\right)}{1+d_{\infty}\left(z w_{1}, z w_{2}\right)}\right\}, \\
& m\left(w_{1}, w_{2}\right)=\min \left\{d_{\infty}\left(z w_{1}, O w_{1}\right), d_{\infty}\left(z w_{2}, O w_{2}\right),\right. \\
& \left.d_{\infty}\left(z w_{1}, O w_{2}\right), d_{\infty}\left(z w_{2}, O w_{1}\right)\right\} .
\end{aligned}
$$

For all $w_{1}, w_{2} \in B(S)$ and all $a \in S$, all $b \in E$. Also, the function $\phi$ is as in Theorem 10.

Then (68) has a unique common solution $w_{0} \in B(S)$.

Proof. First, we prove that the mappings in system (68) satisfy condition (6). Indeed, by using Lemma 19, we have that $\forall w_{1}, w_{2} \in B(S), \forall x \in S$, and we consider

$$
\begin{aligned}
& d_{\infty}\left(O w_{1}, O w_{2}\right) \\
& \quad \leq \sup _{b \in E}\left|F\left(a, b, z\left(w_{1}\right)\right)-F\left(a, b, z\left(w_{2}\right)\right)\right| \\
& \quad \leq \phi\left(M\left(w_{1}, w_{2}\right)\right)+C m\left(w_{1}, w_{2}\right) .
\end{aligned}
$$

Then all the conditions of Theorem 10 are satisfied; hence, system (68) has a unique solution.

\section{Competing Interests}

The authors declare that they have no competing interests.

\section{Authors' Contributions}

The two authors contributed equally to this work. Both authors read and approved the final paper.

\section{References}

[1] L. Cesari, "Functional analysis and periodic solutions of nonlinear differential equations," Contributions to Differential Equations, no. 1, pp. 149-187, 1963.

[2] J. K. Hale, Ordinary Differential Equations, Wiley-Interscience, New York, NY, USA, 1969.

[3] N. Rouche and J. Mawhin, Equations Diferentielles Ordinaires, Vol. I, II, Masson et Cie, Paris, France, 1973.

[4] B. K. Dass and S. Gupta, "An extension of Banach contraction principle through rational expressions," Indian Journal of Pure and Applied Mathematics, vol. 6, no. 12, pp. 1455-1458, 1975.

[5] A. Almeida, A. F. Roldan-Lopez-de-Hierro, and K. Sadarangani, "On a fixed point theorem and its application in dynamic programming," Applicable Analysis and Discrete Mathematics, vol. 9, no. 2, pp. 221-244, 2015.

[6] A. Branciari, "A fixed point theorem of Banach-Caccioppoli type on a class of generalized metric spaces," Publicationes Mathematicae Debrecen, vol. 57, no. 1, pp. 31-37, 2000. 
[7] J. Ahmad, M. Arshad, and C. Vetro, "On a theorem of Khan in a generalized metric space," International Journal of Analysis, vol. 2013, Article ID 852727, 6 pages, 2013.

[8] M. Asadi, E. Karapinar, and A. Kumar, " $\alpha-\psi$-Geraghty contractions on generalized metric spaces," Journal of Inequalities and Applications, vol. 2014, p. 423, 2014.

[9] A. Azam and M. Arshad, "Kannan fixed point theorem on generalized metric spaces," The Journal of Nonlinear Sciences and Its Applications, vol. 1, no. 1, pp. 45-48, 2008.

[10] P. Das and L. K. Dey, "A fixed point theorem in a generalized metric space," Soochow Journal of Mathematics, vol. 33, no. 1, pp. 33-39, 2007.

[11] P. Das and L. K. Dey, "Fixed point of contractive mappings in generalized metric spaces," Mathematica Slovaca, vol. 59, no. 4, pp. 499-504, 2009.

[12] A. Fora, A. Bellour, and A. Al-Bsoul, "Some results in fixed point theory concerning generalized metric spaces," Matematički Vesnik, vol. 61, no. 3, pp. 203-208, 2009.

[13] P. Kumam and N. V. Dung, "Some remarks on generalized metric spaces of Branciari," Sarajevo Journal of Mathematics, vol. 10, no. 23, pp. 209-219, 2014.

[14] Z. Kadelburg and S. Radenovic, "On generalized metric spaces: a survey," TWMS Journal of Pure and Applied Mathematics, vol. 5, no. 1, pp. 3-13, 2014.

[15] Z. Kadelburg and S. Radenović, "Fixed point results in generalized metric spaces without Hausdorff property," Mathematical Sciences, vol. 8, article 125, 2014.

[16] Z. Kadelburg, S. Radenovic, and S. Shukla, "Boyd-Wong and Meir-Keeler type theorems in generalized metric spaces," Journal of Advanced Mathematical Studies, vol. 9, no. 1, pp. 83-93, 2016.

[17] V. L. Rosa and P. Vetro, "Common fixed points for $\alpha-\psi-\phi$ contractions in generalized metric spaces," Nonlinear Analysis: Modelling and Control, vol. 19, no. 1, pp. 43-54, 2014.

[18] I. R. Sarma, J. M. Rao, and S. S. Rao, "Contractions over generalized metric spaces," The Journal of Nonlinear Science and Applications, vol. 2, no. 3, pp. 180-182, 2009.

[19] B. Samet, "A fixed point theorem in a generalized metric space for mappings satisfying a contractive condition of integral type," International Journal of Mathematical Analysis, vol. 3, no. 6, pp. 1265-1271, 2009.

[20] Z. Liu, "Existence theorems of solutions for certain classes of functional equations arising in dynamic programming," Journal of Mathematical Analysis and Applications, vol. 262, no. 2, pp. 529-553, 2001.

[21] S. S. Chang, "Some existence theorems of common and coincidence solutions for a class of functional equations arising in dynamic programming," Applied Mathematics and Mechanics, vol. 12, pp. 31-37, 1991.

[22] S.-S. Chang and Y. H. Ma, "Coupled fixed points for mixed monotone condensing operators and an existence theorem of the solutions for a class of functional equations arising in dynamic programming," Journal of Mathematical Analysis and Applications, vol. 160, no. 2, pp. 468-479, 1991. 


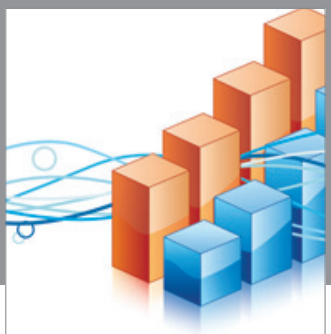

Advances in

Operations Research

vatem alat4

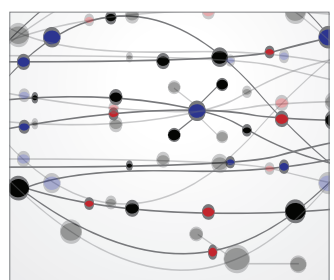

\section{The Scientific} World Journal
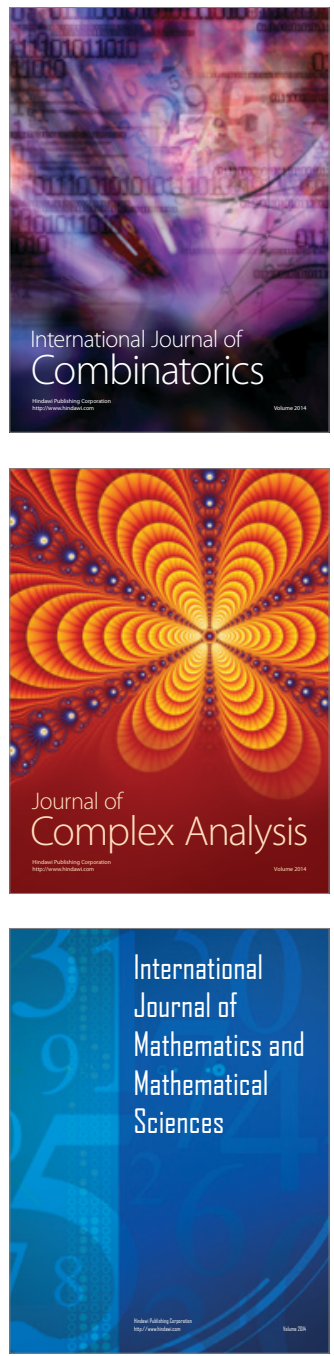
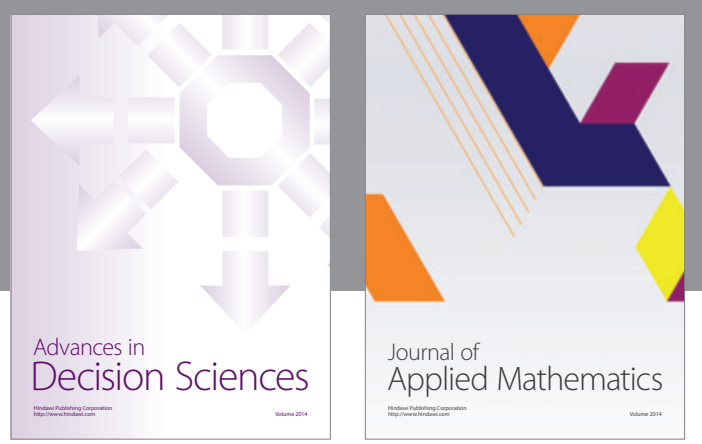

Algebra

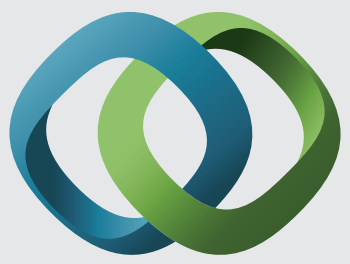

\section{Hindawi}

Submit your manuscripts at

http://www.hindawi.com
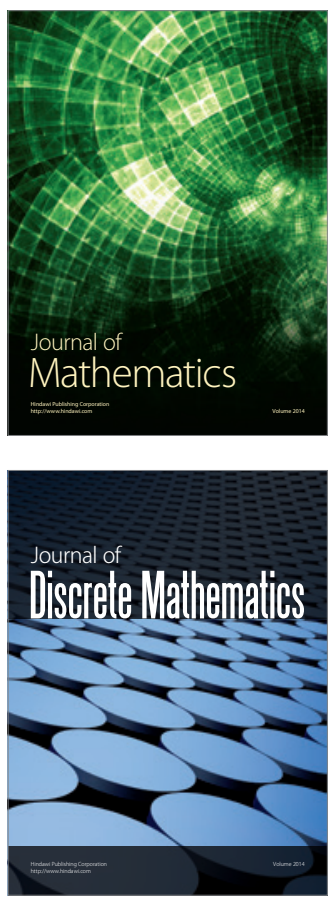

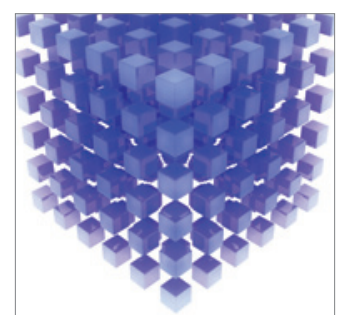

Mathematical Problems in Engineering
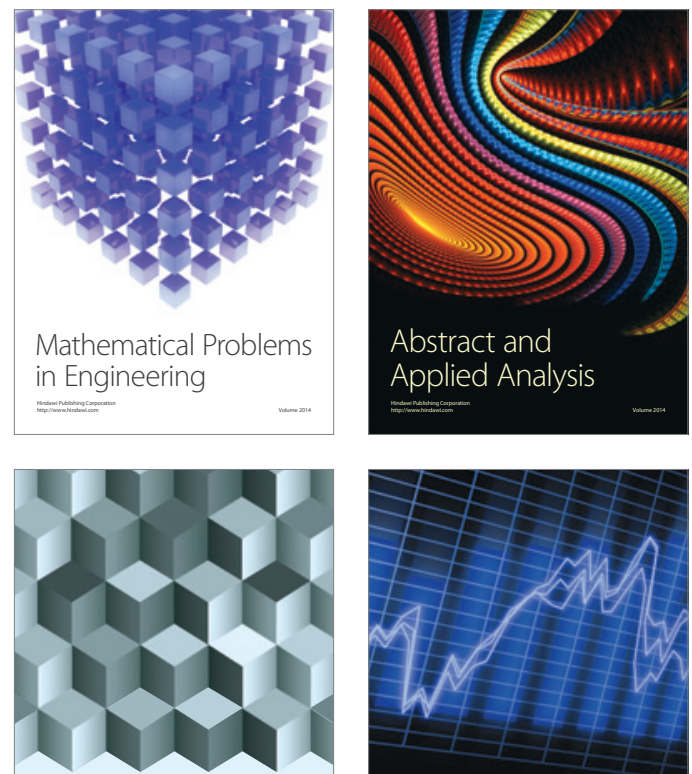

Journal of

Function Spaces

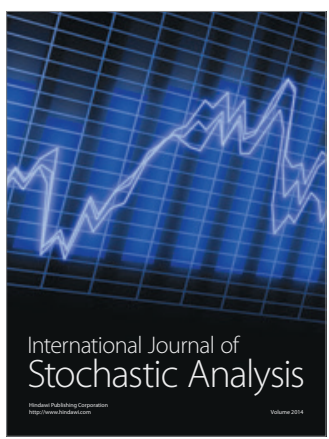

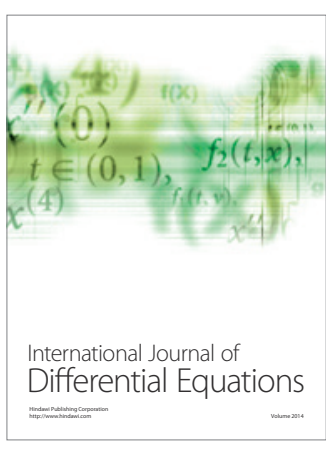
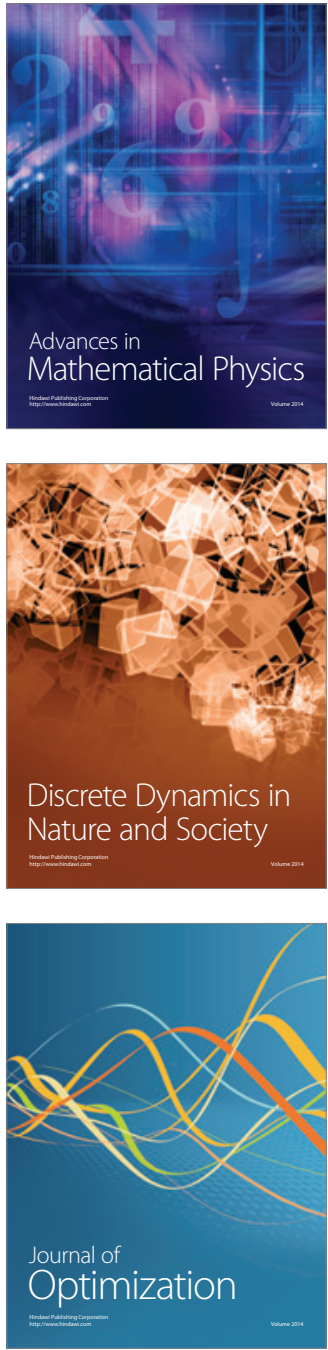